\title{
Ablación de extrasístoles del summit del ventrículo izquierdo en la vena interventricular anterior
}

\section{Catheter ablation of left ventricle summit PVC using anterior interventricular vein approach}

\author{
Luis D. Barja, Juan M. Aboy, Gerson A. Revollo y Emilio A. Logarzo* \\ Servicio de Electrofisiología, Hospital Universitario Austral, Buenos Aires, Argentina
}

\begin{abstract}
Resumen
Se desarrolla el caso de una paciente de 24 años con corazón estructuralmente sano y antecedente de ablación por radiofrecuencia (ARF) de extrasístole ventricular (EV) del tracto de salida del ventrículo izquierdo (VI). Evoluciona a los dos años postablación nuevamente con EV muy frecuentes (> 47,000 en Holter de 24 h) refractarias a múltiples esquema antiarrítmicos y muy sintomática por palpitaciones. Electrocardiograma con EV con morfología izquierda con origen en el summit del VI con ARF epicárdica exitosa a través de la vena interventricular anterior y sin recurrencia arrítmica en la evolución de seis meses.
\end{abstract}

Palabras claves: Ablación por radiofrecuencia. Extrasístoles ventriculares izquierdas. Summit. Vena interventricular anterior. Ablación epicárdica.

\begin{abstract}
A young female patient with normal ejection fraction. History of premature ventricular complex (PVC) radiofrequency ablation located in left ventricular outflow tract. Two years later frequent PVC is observed in a different location (> 47000 in $24 \mathrm{~h}$ Holter). Anti-arrhythmic drugs were used unsuccessfully. PVCs were located in left ventricular summit. Radiofrequency ablation through coronary sinus and anterior interventricular vein was performed. During follow up no recurrence was observed.
\end{abstract}

Keywords: Radiofrequency ablation. Left ventricular extrasystoles. Summit. Anterior interventricular vein. Epicardial ablation.

\section{Introducción}

Según la literatura, alrededor del $12 \%$ de las extrasístoles ventriculares (EV) izquierdas idiopáticas tiene su origen en el summit del ventrículo izquierdo (VI)1. Esta zona está representada por una región triangular epicárdica del tracto de salida del VI con el vértice en la bifurcación entre la arteria coronaria descendente anterior y la circunfleja, y una base formada por un arco que conecta la primera rama perforante septal de la arteria coronaria descendente anterior con la arteria circunfleja. La vena coronaria mayor o vena cardiaca magna (VCM) lo divide lateralmente a la altura de su unión con la vena interventricular anterior (VIA),
Correspondencia:

*Emilio A. Logarzo

E-mail: emiliologarzo@hotmail.com
Disponible en internet: 03-01-2022 Arch Cardiol Mex. 2022;92(1):132-138 www.archivoscardiologia.com 1405-9940 / @ 2020 Instituto Nacional de Cardiología Ignacio Chávez. Publicado por Permanyer. Este es un artículo open access bajo la licencia CC BY-NC-ND (http://creativecommons.org/licenses/by-nc-nd/4.0/). 
segmentándolo de esta manera en una zona accesible y una no accesible (superior), estando esta última realmente alejada del miocardio y es así que algunos focos pueden estar adyacente al epicardio, pudiendo ser esta una vía de acceso para la ablación ${ }^{2}$.

Electrocardiográficamente las extrasístoles ventriculares de la región del summit de VI suelen presentar morfología de bloqueo de rama derecha con eje inferior y desviación a la derecha, con mayor voltaje de la onda $\mathrm{R}$ de DIII, comparada con DII; presencia de QS en DI en alrededor del $30 \%$ de los casos; pérdida brusca de la R en V2 (si bien esto lo comparte con la continuidad mitro-aórtica), comparada con V1 y V3 y relación Q-aVL/Q- aVR > 1.45; con presencia de una deflexión rápida al inicio del QRS (pseudodelta) superior a 34 milisegundos (ms). La importancia de la ablación en dicha zona radica en su considerable frecuencia y las dificultades que plantean los aspectos técnicos de la ablación con catéter en estas estructuras ${ }^{1,2}$.

\section{Caso clínico}

Presentamos una paciente de 24 años. Sin factores de riesgo cardiovascular ni antecedentes familiares. Ablación previa de EV frecuentes del tracto de salida del VI en 2017. Evolucionó asintomática hasta 2019, cuando volvió a presentar palpitaciones muy frecuentes. Holter con > 47,000 EV monomorfas, aisladas, duplas y episodios de taquicardia ventricular no sostenida. Resonancia magnética cardiaca sin fibrosis ni signos de depósitos fibroadiposos, pero con deterioro leve de la función ventricular (fracción de eyección [Fey]: 45\%) asociado a hipocinesia global. El electrocardiograma (ECG) presentaba EV con eje inferior, QS en DI, R alta en V1 con transición sobre V2 y S profunda en V6. El tratamiento con betabloqueantes y amiodarona fue ineficaz, por lo que se programó nueva ablación por radiofrecuencia (ARF).

Los ECG preablación (Fig. 1) mostraron EV con morfología de bloqueo de rama derecha en V1 con $\mathrm{R}$ alta y transición precoz en $\mathrm{V} 2$ y un eje inferior con mayor voltaje de R en DIII comparado a DII con QS en DI y $S$ profunda en V6, pseudodelta de $40 \mathrm{~ms}$, lo cual sugería un posible origen en el summit del $\mathrm{Vl}^{3}$.

Se realizó estudio electrofisiológico y anatomía con sistema de navegación ENSITE VELOCITY 5.0 (St. Jude Medical) de varias estructuras (cúspide coronaria izquierda [CCI], cúspide coronaria derecha [CCD], tracto de salida del ventrículo derecho [TSVD] septal, continuidad mitro-aórtica, seno coronario, la gran vena cardiaca en toda su extensión hasta la unión de la VCM y VIA).
La región del summit, al ser tridimensional y en forma «triangular», está situada en una posición próxima a la arteria coronaria izquierda y a las venas distales. Es posible el mapeo a través de estas estructuras, determinada por sus múltiples relaciones anatómicas con el TSVD, cúspides aórticas, las venas tales como la gran vena aórtica (GDV), VCM y la vena intraventricular anterior y realizar una ablación exitosa desde cualquiera de estas ${ }^{4,5}$.

Durante el procedimiento, para el abordaje retrogrado aórtico se utilizó para el mapeo un catéter de $8 \mathrm{~mm}$ Blazer II de Boston Scientific, catéter de ablación de $4 \mathrm{~mm}$ Blazer de Boston Scientic pediátrico de $6 \mathrm{Fr}$ en seno coronario y catéteres decapolares en haz de His y TSVD.

Se realizó mapeo de la región septal del TSVD, donde se observaba una señal endocavitaria ventricular local tardía después del QRS y del seno coronario distal y poca concordancia con las EV en el pace mapping.

Se observó que el catéter multipolar en zona distal próxima a la unión de la VCM y la VIA presentaba una precocidad adecuada sugiriendo la cercanía de esa zona de abordaje respecto al foco de la EV. Al mapear distalmente con el catéter de ablación introduciendo el catéter de mapeo a través de la vena cardiaca anterior se observó mayor precocidad (Figs. 2 y 3).

El mapa de activación temporal con mapeo de zonas cercanas como las $\mathrm{CCl}$, la zona mitro-aórtica y la gran vena cardiaca, evidenció una precocidad de $-20 \mathrm{~ms}$ evidenciando cercanía, pero no lo suficiente (Fig. 4). Al mapear la vena interventricular anterior la precocidad fue más importante, coincidente con el registro del polígrafo que mostró una señal ventricular local de -68 ms que precedía al inicio del QRS (Figs. 2 y 3), con una concordancia en el pace mapping de 12/12 (Fig. 5) y un mapa LAT (local activation time) que evidenció la misma zona de precocidad.

Ante la posibilidad de realizar aplicaciones dentro de la vena interventricular anterior por su probable cercanía con la arteria circunfleja, arterias septales o la bifurcación del tronco de la arteria coronaria izquierda, se realizó disparo en el tronco de la coronaria izquierda previo a la ablación evidenciando la cercanía de $5 \mathrm{~mm}$ aproximadamente, resultado que nos permitió proseguir, ya que una ablación cercana a estas estructuras genera riesgo de lesión de estas.

Se decidió ablación con un monitoreo estricto de la presión arterial invasiva y del ECG con un catéter de $4 \mathrm{~mm}$ Blazer de BSC pediátrico de $6 \mathrm{Fr}$ dado su menor diámetro (actualmente dichas ablaciones las realizamos con catéter irrigado y fuerza de contacto) a $45 \stackrel{\circ}{\circ}$ 


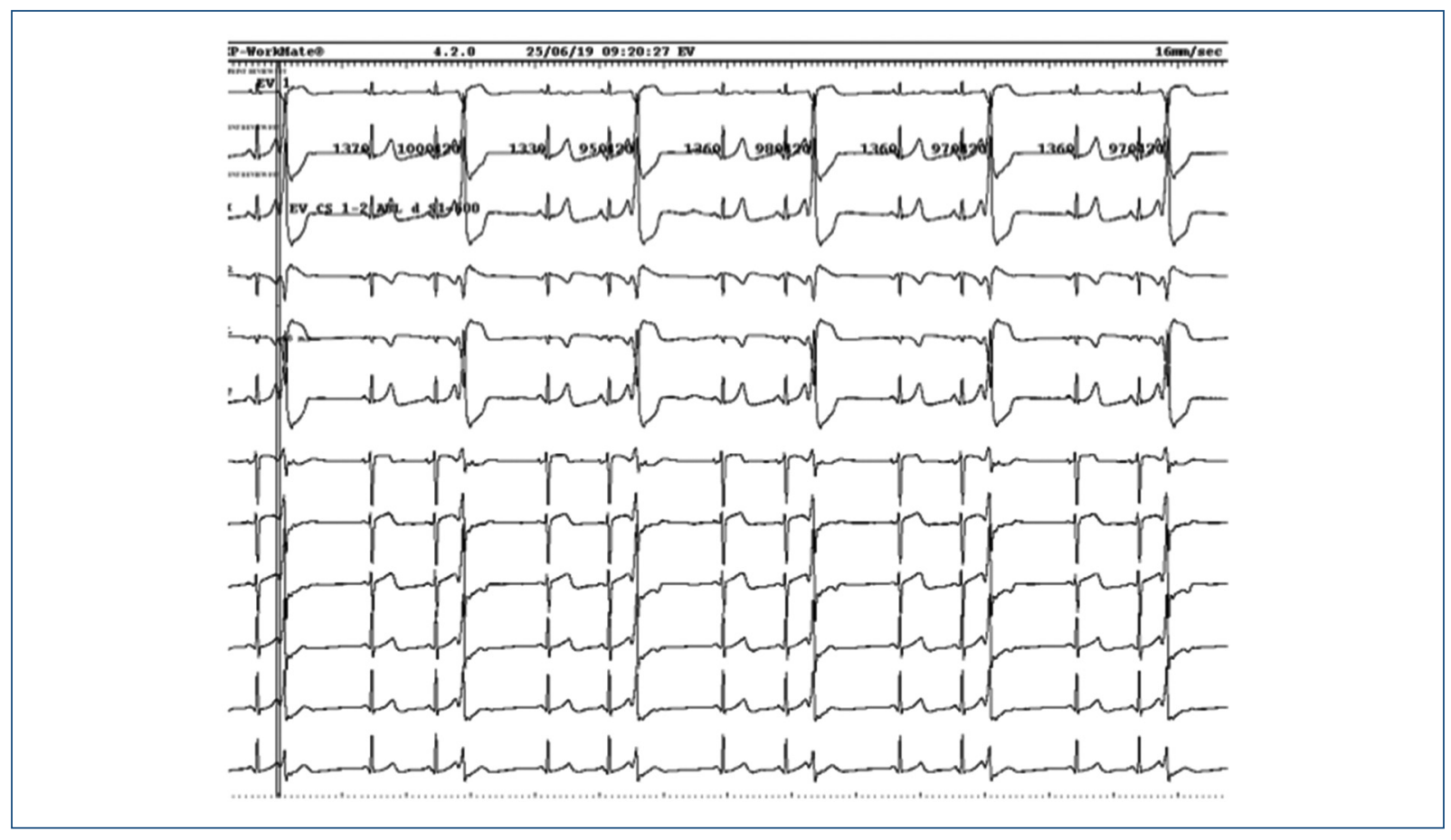

Figura 1. Electrocardiograma basal intraprocedimiento.

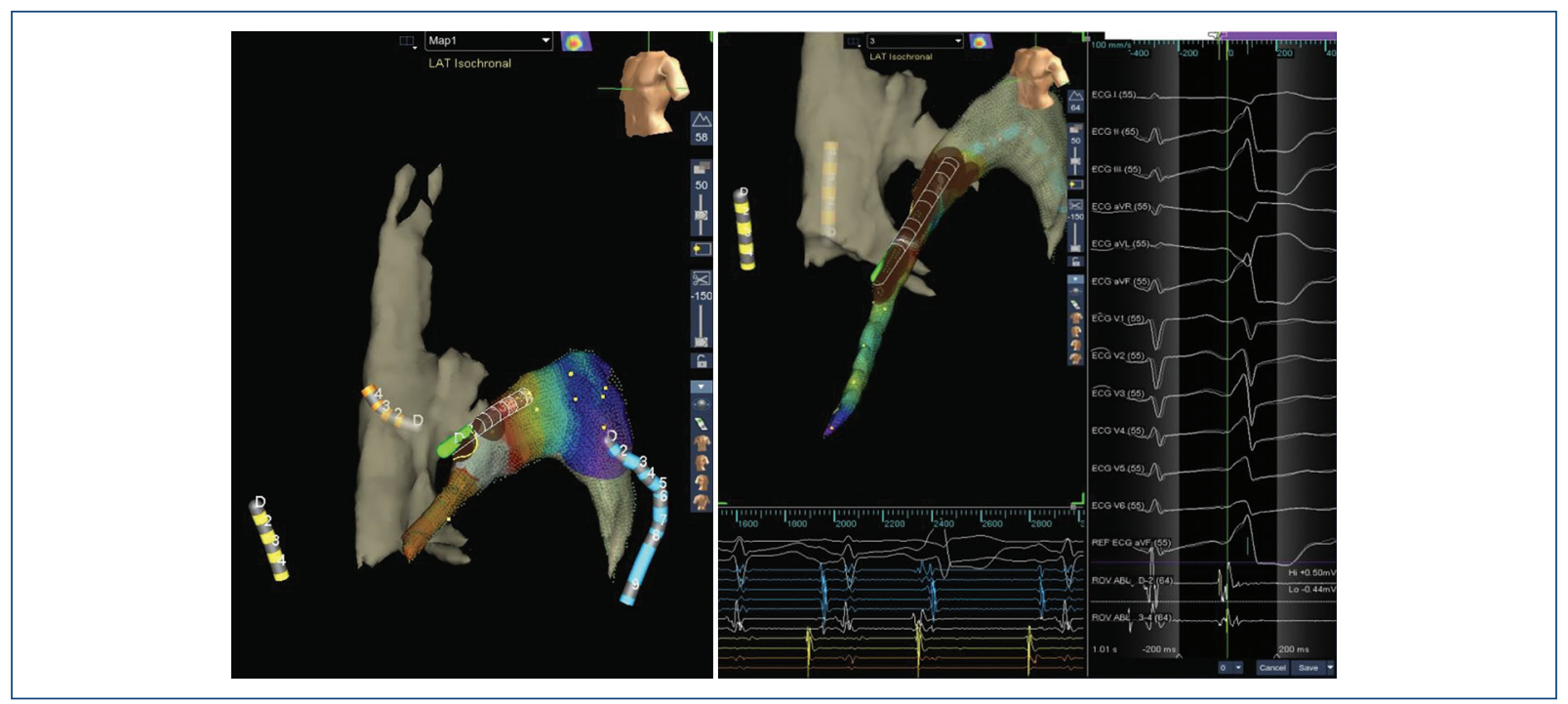

Figura 2. Mapeo en vena interventricular anterior.

de temperatura y $30 \mathrm{~W}$ con incrementos progresivos de temperatura hasta alcanzar los $55^{\circ} \mathrm{C}$ apagando el límite de impedancia por corte frecuente por esta, llegando a la temperatura deseada logrando aplicaciones de hasta $20 \mathrm{~W}$ con una duración de hasta 90 segundos totales en la tercera aplicación. Se logró con éxito la ablación sin reproducibilidad de la arritmia (Fig. 6) y excelente evolución posterior (ecocardiograma Doppler y resonancia cardiaca al mes postablación con Fey $60 \%$ sin trastornos de la motilidad y Holter sin arritmia ventricular), lo que confirma que estábamos ante un cuadro de taquicardio-miopatía. 


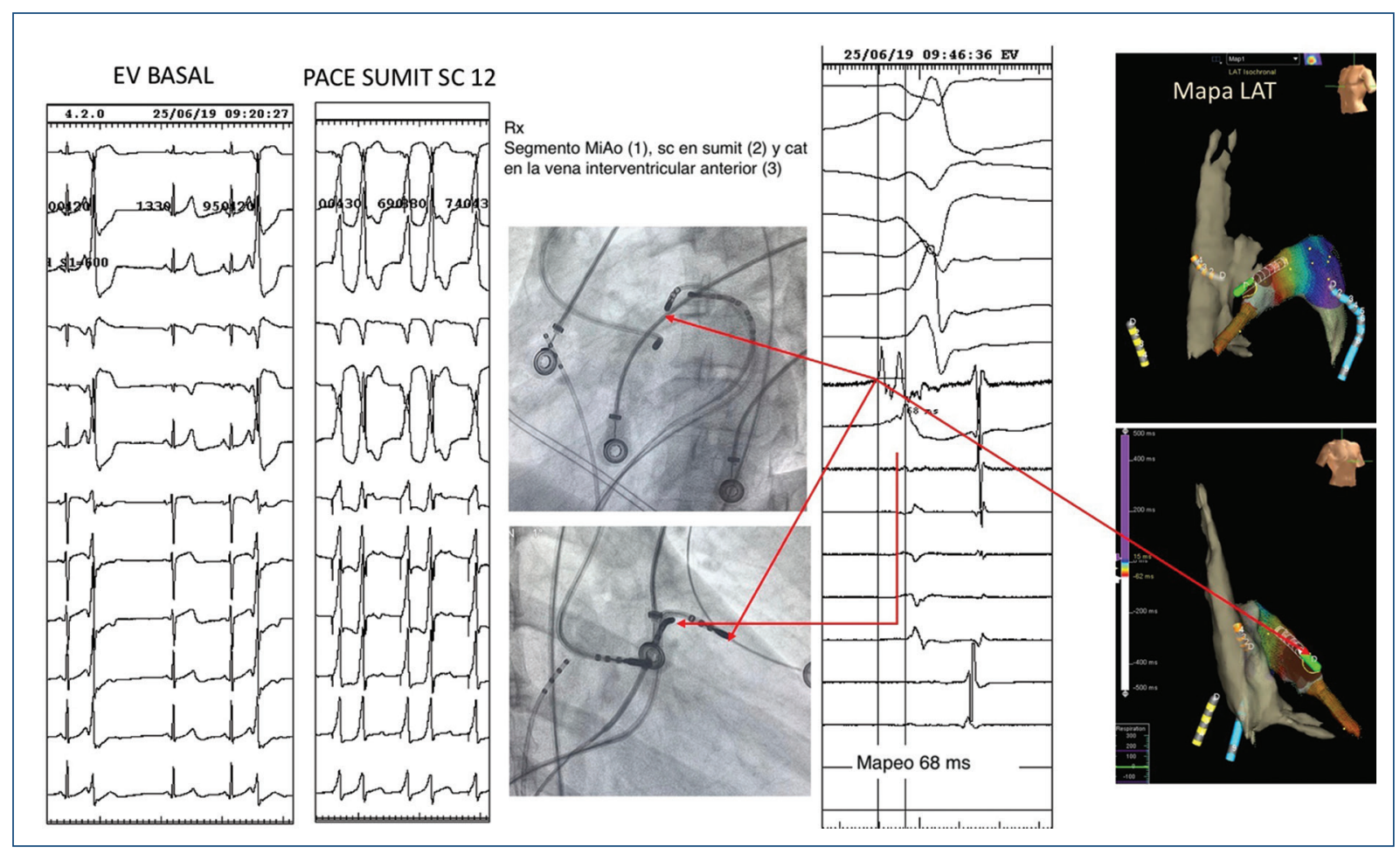

Figura 3. A la derecha de la imagen se observa el electrocardiograma de 12 derivaciones con la extrasístole ventricular con posible ubicación en summit de ventrículo izquierdo. En la imagen de al lado se observa el momento de la realización del pace mapping con una concordancia 12/12. En la imagen central se observa una imagen radioscópica en proyección OAI (arriba) y OAD (abajo) donde se ve la posición de los catéteres dentro del seno coronario. La imagen a la derecha muestra la precocidad obtenida previa a la aplicación de radiofrecuencia de $68 \mathrm{~ms}$. En la última imagen a la derecha, el mapa tridimensional muestra el sitio donde se realizó la ablación por radiofrecuencia en forma exitosa. EC: extrasístoles ventriculares; RX: radiografía de tórax; sc: seno coronario; MiAo: continuidad mitro-aórtica; cat: catéter; LAT: local activation time.

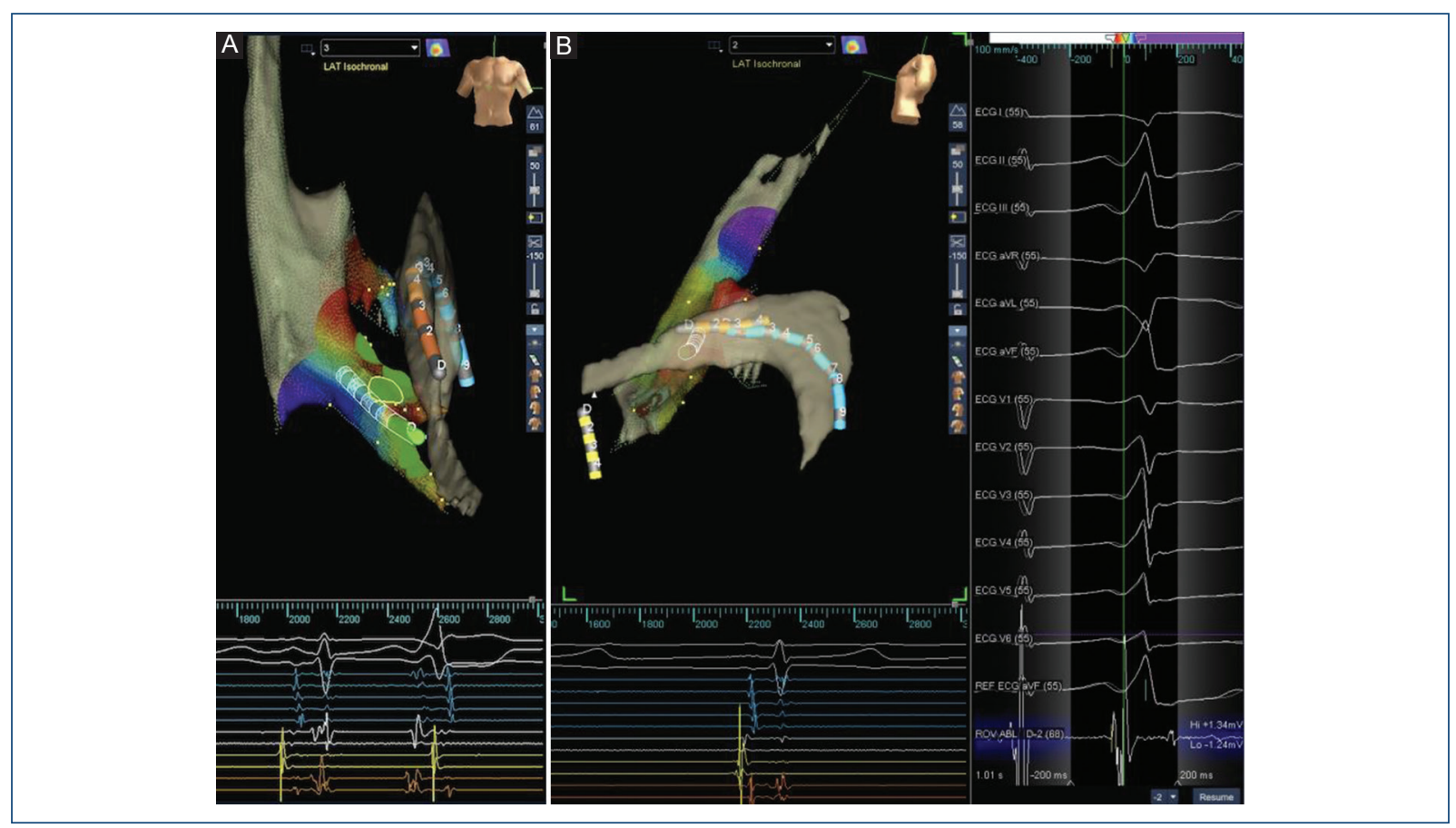

Figura 4. A: mapeo en zona mitro-aórtica. B: mapeo en cúspide coronaria izquierda. 


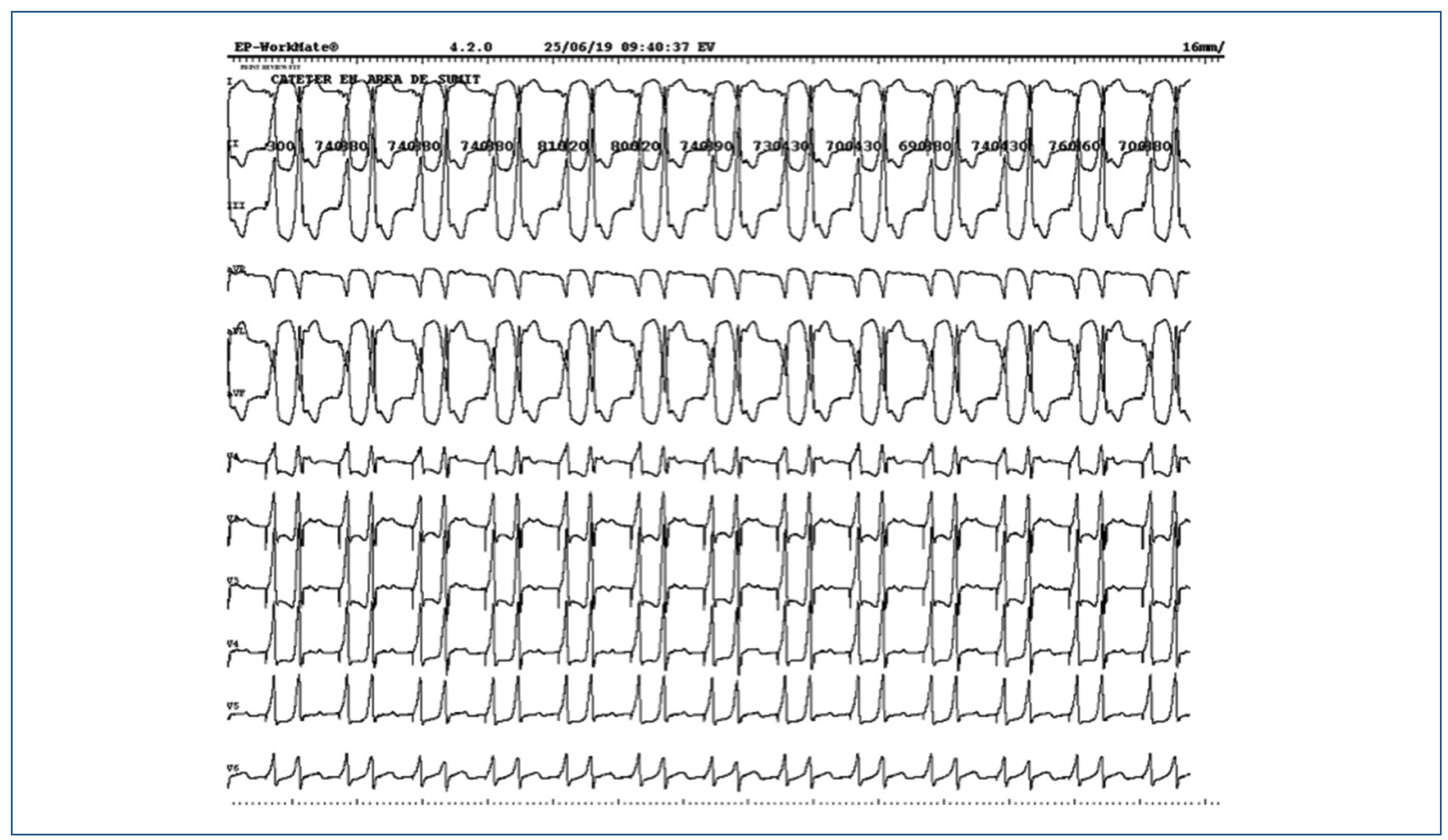

Figura 5. Topoestimulación con concordancia 100\% en región del summit.

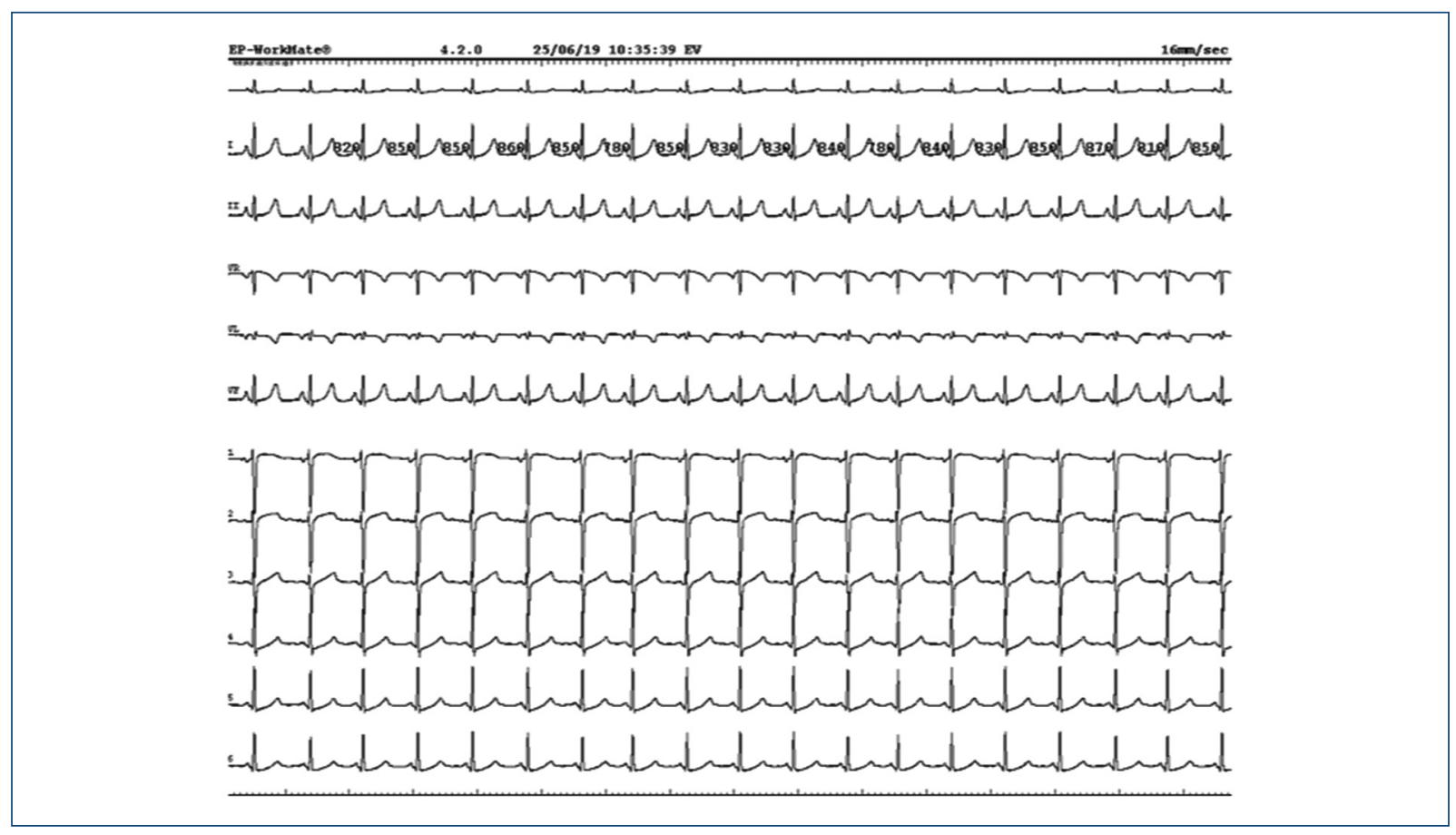

Figura 6. Electrocardiograma postablación.

\section{Discusión}

Las ablaciones del summit son un reto para los inter- vencionistas, básicamente por su estructura

tridimensional, la posibilidad de abordaje de distintas zonas, muchas de ellas epicárdicas, la cercanía de estructuras nobles como las arterias coronarias y accesos complicados que requieren una sistemática 
protocolaria para ir evaluando y descartando esas zonas $^{6,7}$.

Requiere el mapeo de zonas como las cúspides coronarias, sobre todo la izquierda, la zona epicárdica de la gran vena cardiaca, muchas veces el ingreso retrógrado aórtico subvalvular y evaluar la continuidad mitro-aórtica e incluso también estructuras del TSVD en su porción septal o arteria pulmonar 8 .

Conociendo las limitaciones de la zona del summit, que al tener una estructura superior de no contacto (no accesible) con el miocardio la hace no factible de su ablación.

La ablación a través de las estructuras venosas se ha realizado con éxito en diversas situación tales como ablación de Wolf Parkinson White (WPW), desfragmentación del seno coronario en la fibrilación auricular y ablaciones epicárdicas de taquicardias auriculares, entre otras. La técnica y la seguridad están comprobados sobre todo hoy con la tecnología de catéteres irrigados y fuerza de contacto.

Tanto el acceso, el mapeo, como la ablación en estas estructuras venosas y epicárdicas son un reto, ya que dependerá de factores como sus diámetros, angulaciones, válvulas, donde muchas veces solo utilizando catéteres muy finos que puedan acceder. En dichas zonas se han encontrado potenciales de baja amplitud que pueden servir para guiar y predecir por su precocidad los focos aledaños de extrasístoles ${ }^{10}$.

Lo mismo sucede con la ablación dentro de la VIA con la abrupta concentración de energía liberada, el corte constante por la alta impedancia (donde la mayoría de las veces hay que anular esta herramienta de medición y corte), la cercanía de estructuras arteriales como la arteria descendente anterior y circunfleja (por lo que es condición necesaria realizar la anatomía coronaria para su ablación) o a la imposibilidad de alcanzar una potencia suficiente y una lesión en profundidad.

En nuestro caso se realizó un disparo en el tronco de la coronaria izquierda antes del momento de la ablación con el catéter en posición demostrando la no cercanía de estructuras arteriales respecto al dipolo distal de mapeo. Esta imagen no ha sido grabada, dado que era un equipo de radiología para electrofisiología sin posibilidad de grabación de una coronariografía.

En algunas oportunidades se requieren accesos distintos donde no se pueden diferenciar el mejor de los sitios de mapeo, siendo muy próximos entre sí, a veces en esos casos parece lógico comenzar por las zonas donde uno tenga menor riesgo y mayor experiencia dejando para procedimientos posteriores, por ejemplo, zonas que requieran mayor complejidad como el abordaje epicárdico subxifoideo demostrado por el grupo del Dr.Sosa, et al.11,12.

En otros casos, si coincide el foco epicárdico de la EV con sitios donde estas estructuras venosas discurren, y puedan estar cercanas, se podrá utilizar estos abordajes para aplicaciones de energía eficaces para ablacionar dichas arritmias, pueden entonces ser contempladas estas estructuras como metodología en el abordaje.

Otra singularidad que se evidenció en este caso es la mejoría de la función ventricular postablación producida por su «arritmo-miopatía» sugerida como similar a la «taquicardio-miopatía». Si bien no se trata en este caso de frecuencias cardiacas altas, sino de la densidad de las EV cercana al $24 \%$ del total ${ }^{13,14}$, los sitios de origen y su ligadurat5-17.

De manera tal cuando la ablación no es eficaz desde el endocardio o desde estructuras venosas como vimos, puede contemplarse el empleo de un abordaje epicárdico percutáneo ${ }^{18,19}$.

\section{Conclusión}

El mapeo y la ARF a través de estructuras venosas cuando los focos se originan cercanos a ellas puede ser una opción de abordaje técnicamente factible. Si bien habrá detalles para evitar complicaciones, esto dependerá de un correcto análisis anatómico. En este caso se pudo abordar el mapeo y la ablación vía seno coronario a través de la gran vena cardiaca hasta alcanzar la vena interventricular anterior, que fue factible debido a sus características anatómicas y el origen de la EV se encontraba muy próximo. Se logró incluso el beneficio de mejorar su «arritmo-miopatía» asociada.

\section{Financiamiento}

$$
\text { Ninguno. }
$$

\section{Conflicto de intereses}

Los autores no presentan conflictos de intereses.

\section{Responsabilidades éticas}

Protección de personas y animales. Los autores declaran que para esta investigación no se han realizado experimentos en seres humanos ni en animales. 
Confidencialidad de los datos. Los autores declaran que han seguido los protocolos de su centro de trabajo sobre la publicación de datos de pacientes.

Derecho a la privacidad y consentimiento informado. Los autores declaran que en este artículo no aparecen datos de pacientes.

\section{Bibliografía}

1. Enriquez A, Malavassi F, Saenz LC, Supple G, Santangeli P, Marchlinski FE, et al. How to map and ablate left ventricular summit arrhythmias. Heart Rhythm. 2017;14(1):141-8.

2. Yamada T, McElderry HT, Doppalapudi H, Okada T, Murakami Y, Yoshida $Y$, et al. Idiopathic ventricular arrhythmias originating from the left ventricular summit: Anatomic concepts relevant to ablation. Circ Arrhythm Electrophysiol. 2010;3(6):616-23.

3. Berruezo A, Mont L, Nava S, Chueca E, Bartholomay E, Brugada J. Electrocardiographic recognition of the epicardial origin of ventricular tachycardias. Circulation. 2004;109(15):1842-7.

4. Jauregui Abularach ME, Campos B, Park K-M, Tschabrunn CM Frankel DS, Park RE, et al. Ablation of ventricular arrhythmias arising near the anterior epicardial veins from the left sinus of Valsalva region: ECG features, anatomic distance, and outcome. Heart Rhythm. 2012;9(6):865-73.

5. Lin C-Y, Chung F-P, Lin Y-J, Chong E, Chang S-L, Lo L-W, et al. Radiofrequency catheter ablation of ventricular arrhythmias originating from the continuum between the aortic sinus of Valsalva and the left ventricula summit: Electrocardiographic characteristics and correlative anatomy. Heart Rhythm. 2016;13(1):111-21.

6. Cronin EM, Bogun FM, Maury P, Peichl P, Chen M, Namboodiri N, et al. 2019 HRS/EHRA/APHRS/LAHRS expert consensus statement on catheter ablation of ventricular arrhythmias. Europace. 2019;21(8):1143-4.

7. Dukkipati SR, Choudry S, Koruth JS, Miller MA, Whang W, Reddy VY. Catheter ablation of ventricular tachycardia in structurally normal hearts. J Am Coll Cardiol. 2017;70(23):2909-23.
8. Prystowsky EN, Padanilam BJ, Joshi S, Fogel RI. Ventricular arrhythmias in the absence of structural heart disease. J Am Coll Cardiol. 2012;59(20):1733-44.

9. Shimizu W. Arrhythmias originating from the right ventricular outflow tract: How to distinguish "malignant" from "benign"? Heart Rhythm. 2009;6(10):1507-11.

10. Reithmann C, Fiek M, Hahnefeld A, Ulbrich M, Steinbeck G. Recording of low-amplitude diastolic electrograms through the coronary veins: a guide for epicardial ventricular tachycardia ablation. Europace. 2012;14(6):865-71.

11. Sosa E, Scanavacca M, D'Avila A, Piccioni J, Sanchez O, Velarde JL, et al. Endocardial and epicardial ablation guided by nonsurgical transthoracic epicardial mapping to treat recurrent ventricular tachycardia. J Cardiovasc Electrophysiol. 1998:9(3):229-39.

12. Della Bella P, Brugada J, Zeppenfeld K, Merino J, Neuzil P, Maury P, et al. Epicardial ablation for ventricular tachycardia: A European multicenter study. Circ Arrhythm Electrophysiol. 2011;4(5):653-9.

13. Baman TS, Lange DC, Ilg KJ, Gupta SK, Liu T-Y, Alguire C, et al. Relationship between burden of premature ventricular complexes and left ventricular function. Heart Rhythm. 2010;7(7):865-9.

14. Hasdemir C, Ulucan C, Yavuzgil O, Yuksel A, Kartal Y, Simsek E, et al. Tachycardia-induced cardiomyopathy in patients with idiopathic ventricular arrhythmias: The incidence, clinical and electrophysiologic characteristics, and the predictors. J Cardiovasc Electrophysiol. 2011;22(6):663-8.

15. Kuroki K, Tada $\mathrm{H}$, Seo $Y$, Ishizu T, Igawa M, Yamasaki $H$, et al. Prediction and mechanism of frequent ventricular premature contractions related to haemodynamic deterioration. Eur J Heart Fail. 2012;14(10):1112-20.

16. Park K-M, Im SI, Park S-J, Kim JS, On YK. Risk factor algorithm used to predict frequent premature ventricular contraction-induced cardiomyopathy. Int J Cardiol. 2017;233:37-42.

17. Bas HD, Baser K, Hoyt J, Yokokawa M, LaBounty T, Morady F, et al. Effect of circadian variability in frequency of premature ventricular complexes on left ventricular function. Heart Rhythm. 2016;13(1):98-102.

18. Santangeli $P$, Marchlinski FE, Zado ES, Benhayon D, Hutchinson MD, Lin D, et al. Percutaneous epicardial ablation of ventricular arrhythmias arising from the left ventricular summit: Outcomes and electrocardiogram correlates of success. Circ Arrhythm Electrophysiol. 2015;8(2):337-43.

19. Gil-Ortega I, Pedrote-Martínez A, Fontenla-Cerezuela A. Spanish Catheter Ablation Registry. $14^{\text {th }}$ Official Report of the Spanish Society of Cardiology Working Group on Electrophysiology and Arrhythmias (2014). Rev Esp Cardiol (Engl Ed). 2015;68(12):1127-37. 